\title{
Treating Early Delayed Gastric Tube Emptying after Esophagectomy with Pneumatic Pyloric Dilation
}

\author{
Alexander Mertens $s^{a, b}$ Jan Gooszen ${ }^{a}$ Paul Fockens ${ }^{c}$ Rogier Voermans ${ }^{c}$ \\ Suzanne Gisbertz ${ }^{\mathrm{a}} \quad$ Arjan Bredenoord $^{c}$ Mark Ivo van Berge Henegouwen ${ }^{\mathrm{a}}$ \\ aDepartment of Surgery, Amsterdam University Medical Centers, location AMC, University of Amsterdam, Cancer \\ Center Amsterdam, Amsterdam, The Netherlands; ${ }^{b}$ Robotics and Mechatronics, University of Twente, Enschede, \\ The Netherlands; ' Department of Gastroenterology \& Hepatology, Amsterdam University Medical Centers, location \\ AMC, University of Amsterdam, Amsterdam Gastroenterology \& Metabolism, Amsterdam, The Netherlands
}

\section{Keywords}

Esophageal cancer surgery - Gastric tube reconstruction . Gastroparesis · Delayed conduit emptying · Pyloric dilation

\begin{abstract}
Introduction: Endoscopic pneumatic pyloric balloon dilation is a treatment option for early postoperative delayed gastric tube emptying following esophageal resection. This study aimed to determine the safety and effectiveness of endoscopic balloon dilation. Methods: Between 2015 and 2018, patients with delayed gastric emptying 8-10 days after esophageal resection with gastric tube reconstruction due to esophageal carcinoma were considered for inclusion. Inclusion criteria were $\geq 1$ of the following: nasogastric tube production $\geq 500 \mathrm{~mL} / 24 \mathrm{~h}, \geq 300 \mathrm{~mL}$ gastric retention, $\geq 50 \%$ gastric tube dilatation on $\mathrm{X}$-ray, or nasogastric tube replacement. Patients were excluded on evidence of anastomotic leakage or reintervention. Success was defined as the ability to expand intake without needing to replace the nasogastric tube. Dilation was performed using a $30-\mathrm{mm}$ Rigiflex balloon. Results: Fifteen patients underwent pyloric dilation, 12 according to the study protocol. Treatment was performed at a median of 12 days (IQR 9-15) postoperatively. Success was achieved in 58\%. At 3 months, 8 patients progressed to
\end{abstract}

karger@karger.com www.karger.com/dsu

Karger"

BOPEN ACCESS
(C) 2021 The Author(s)

Published by S. Karger AG, Basel

This is an Open Access article licensed under the Creative Commons Attribution-NonCommercial-4.0 International License (CC BY-NC) (http://www.karger.com/Services/OpenAccessLicense), applicable to the online version of the article only. Usage and distribution for commercial purposes requires written permission. exclusively oral intake. The remaining 4 patients had supplementary nightly enteral tube feeding. There were no adverse events. Conclusion: Endoscopic balloon dilation of the pylorus is a safe, feasible therapy for early postoperative delayed gastric emptying. With a success rate of $58 \%$, a clinical trial is a necessary next step.

(c) 2021 The Author(s)

Published by S. Karger AG, Basel

\section{Introduction}

Delayed gastric emptying affects up to half of the patients with a gastric tube reconstruction after an esophagectomy and can lead to a severely impaired quality of life [1-3]. Various definitions are used in scientific publications and literature, with an incidence varying between 11 and 50\% [3-7]. Delayed gastric emptying can occur early or late, with the cutoff between these commonly set at 3 months after surgery.

A range of treatments exist, with varying success rates. Prophylactic intraoperative treatments like pyloric digital fracture [8] have shown promising results but could possi-

Alexander Mertens and Jan Gooszen contributed equally to this work.

Correspondence to:

Alexander Mertens, dsu@sandermertens.nl

Mark Ivo Van Berge Henegouwen, m.i.vanbergehenegouwen@ amsterdamumc.nl 
Table 1. Definition of early delayed gastric emptying used for this study

One or more of the following, 8-10 days after the initial surgery

- Inability of removing the nasogastric tube because of persistent production of the nasogastric tube of $>500 \mathrm{~mL}$ per $24 \mathrm{~h}$

- Retention of $>300 \mathrm{~mL}$ after closure of the nasogastric tube for $4 \mathrm{~h}$

- A $>50 \%$ dilatation of the gastric tube on postoperative X-ray, compared with the X-ray directly postoperatively

- Replacement of the nasogastric tube (due to nausea, vomiting, thoracic pain, or liquid level in the gastric tube on chest X-ray)

bly lead to complications. Intraoperative pyloromyotomy or pyloroplasty have shown mixed results and, in our experience, lead to a longer procedure $[9,10]$. It is possible to perform pyloric balloon dilation intraoperatively, but this is not extensively researched and warrants an extra endoscopy during the procedure [11]. Botox injections can be administered intraoperatively although the results are temporary, and this also warrants an intraoperative endoscopy [12]. Prophylactic interventions can lead to overtreatment since the vast majority of patients will never experience this complication, even without intervention [4-6].

Postoperatively, there are a variety of treatment options. Treatment with prokinetic medication such as erythromycin or D2-receptor antagonists like domperidone can be effective but may require continued treatment [13]. Endoscopic treatment with Botox has been proposed as a less-invasive (temporary) alternative, but studies show mixed results $[14,15]$.

Postoperative endoscopic balloon dilation of the pylorus is increasingly performed and has no reported significant adverse events. However, this is only well described in pre- or intraoperative treatment or in patients treated during outpatient care [7, 16-19]. This study aimed to evaluate the efficacy and safety of endoscopic pneumatic pyloric balloon dilation in the early postoperative in-hospital phase in patients with delayed gastric emptying after esophagectomy with gastric tube reconstruction.

\section{Materials and Methods}

This study was conducted in the Amsterdam University Medical Center, location AMC, in Amsterdam. The study was conducted in accordance with the Declaration of Helsinki (as revised in 2013). The study was approved by the Medical Ethics Review Committee of the Amsterdam University Medical Center (No. W16_134 \# 16.155), and informed consent requirement was waived for this study.

\section{Patient Characteristics}

To assess the effectiveness of endoscopic pneumatic dilation of the pylorus in the early postoperative phase, all patients 18 years of age or older suffering from early postoperative delayed gastric emptying 8-10 days after esophagectomy for cancer with a gastric tube reconstruction without medicinal treatment (e.g., motilityenhancing medication) were evaluated for inclusion. This timeframe of POD 8-10 was chosen since an earlier intervention might cause an increased risk of anastomotic complications. The definition of early postoperative delayed gastric emptying as used in this study is summarized in Table 1.

Inclusion criteria were nasogastric tube production $>500$ $\mathrm{mL} / 24 \mathrm{~h}$, gastric retention of $>300 \mathrm{~mL}$ as measured by clamping the NGT for $3 \mathrm{~h}$, over $50 \%$ gastric tube dilatation on AP chest Xray, or the need for replacement of a nasogastric tube at any point after removal. Patients with an anastomotic leakage, anamnestic evidence of an anastomotic stricture, or other surgery-related postoperative morbidity requiring reintervention or intensive care unit admittance were excluded. Additionally, patients not treated according to the study protocol described in this study were excluded.

\section{Procedure}

Since patients were included after the esophageal resection, this study did not standardize the surgical technique. All patients received a nasogastric tube and a jejunostomy as standard practice. The different procedures performed in our study cohort are summarized in Table 2. Patients were planned for in-hospital pyloric dilation by one of the available expert interventional endoscopists at the Amsterdam UMC, location AMC, as soon as possible after inclusion. The study protocol is based on our standard practice and was followed in all patients in the cohort. Twenty-four hours prior to the pyloric dilation, patients were kept nil per mouth with suction applied to the nasogastric tube. During dilation, the nasogastric tube was removed. Procedures were performed under conscious or deep sedation. A therapeutic gastroscope was used to identify the pylorus and to exclude an anastomotic stricture. After introduction of a guidewire in the duodenum, the location of the pylorus was identified using fluoroscopy combined with the location of the endoscope. After removing the gastroscope, a $30-\mathrm{mm}$ diameter pneumatic balloon (Rigiflex; Boston Scientific ${ }^{\mathbb{B}}$ ) was introduced over the guidewire until half of the balloon passed the pylorus. The balloon was dilated up to $5 \mathrm{psi}$ for $1 \mathrm{~min}$, followed by 8 psi for another minute.

On the same day as the intervention, patients were started on a clear liquid diet. After this, the diet was expanded to liquid diet, followed by a solid diet according to protocol. On day 1 and 2 following the intervention, a chest X-ray was made. A nasogastric tube was reinserted in case of $>50 \%$ dilatation of the gastric tube compared to before the procedure, liquid level in the gastric tube, or presence of clinical symptoms of nausea, vomiting, or thoracic pain. Patients were then placed on clear fluids only. The nasogastric tube was removed when production was $<300 \mathrm{~mL}$ per $24 \mathrm{~h}$, as 
measured after clamping the NGT for $3 \mathrm{~h}$. All patients were monitored in the outpatient department following the Dutch guidelines for esophageal cancer, including consultation by a dietician until oral intake was sufficient.

\section{Outcomes}

The primary outcome was clinical treatment success. Clinical treatment success was defined as the ability to expand dietary intake at any rate, without the need to replace the nasogastric tube. Clinical treatment failure was defined as the necessity to replace the nasogastric tube according to the definitions described in the procedure section. Secondary outcomes were technical success of the pyloric dilatation (the ability to complete the endoscopic pneumatic balloon dilation without complications), dietary intake at discharge, at 3 and 6 months, hospital stay, complication rate, subjective reflux at 6-week follow-up, and reinterventions after the pyloric dilation.

\section{Statistical Analysis}

Statistical analysis was performed using SPSS Statistics Version 25.0 (Armonk, NY, USA). Categorical data are presented as absolute numbers and/or percentages. Continuous data are presented as means with standard deviation (SD) for normally distributed data or medians and interquartile ranges (IQR) when not normally distributed.

\section{Results}

In the period between November 2015 and October 2018, 239 esophageal resections for cancer with gastric tube reconstruction were performed. Fifteen (6\%) patients showed evidence of early postoperative delayed gastric emptying according to the definition in Table 1. All 15 patients met the inclusion criteria. No patients underwent intraoperative pyloric fracture. Three patients underwent balloon dilation using an 18-mm balloon instead of the $30-\mathrm{mm}$ balloon described in the study protocol due to preference of the endoscopist and were therefore excluded from further analysis. Table 2 shows the baseline characteristics.

There were no intraoperative complications in any of the patients. Postoperatively, 1 patient suffered from a cerebral vascular infarct directly after surgery requiring intra-arterial thrombectomy with complete recovery. Another patient suffered from pneumonia 3 days postoperatively, successfully treated by antibiotic therapy. The 90-day mortality rate was zero.

\section{Pyloric Balloon Dilation}

Table 3 outlines the outcomes of early endoscopic pneumatic balloon dilation of the pylorus. The balloon dilation was clinically successful in 7 patients (58\%), as defined in the study protocol. Five patients (42\%) required replacement of the nasogastric tube: $2(17 \%)$ due to nau-
Table 2. Characteristics of patients included in the study

\begin{tabular}{|c|c|}
\hline Variable & $\begin{array}{l}\text { Outcome } \\
(N=12)\end{array}$ \\
\hline Age, mean (SD), years & $61(8.9)$ \\
\hline \multicolumn{2}{|l|}{ Gender, $N(\%)$} \\
\hline Male & $10(83)$ \\
\hline ASA, median (IQR) & $2(1-2)$ \\
\hline Preop BMI, mean (SD), kg/m² & $25.7(4.1)$ \\
\hline \multicolumn{2}{|l|}{ Comorbidities, $N(\%)$} \\
\hline Diabetes & $0(-)$ \\
\hline Cardiovascular & $7(58)$ \\
\hline Pulmonary & $1(8)$ \\
\hline \multicolumn{2}{|l|}{ Neoadjuvant treatment, $N(\%)$} \\
\hline Chemotherapy & $1(8)$ \\
\hline Chemoradiotherapy & $10(83)$ \\
\hline None & $1(8)$ \\
\hline \multicolumn{2}{|l|}{ Tumor type, $N(\%)$} \\
\hline Adenocarcinoma & $8(67)$ \\
\hline Squamous cell ca. & $3(25)$ \\
\hline Others & $1(8)$ \\
\hline \multicolumn{2}{|l|}{ Tumor location, $N(\%)$} \\
\hline Mid esophagus & $1(8)$ \\
\hline Distal esophagus & $8(67)$ \\
\hline GE-junction & $2(17)$ \\
\hline Cardia & $1(8)$ \\
\hline \multicolumn{2}{|l|}{ Reconstruction, $N(\%)$} \\
\hline Gastric tube & $12(100)$ \\
\hline Conversion, $N(\%)$ & $0(-)$ \\
\hline \multicolumn{2}{|l|}{ Surgical approach, $N(\%)$} \\
\hline MI Ivor-Lewis & $10(83)$ \\
\hline MI McKeown & $1(8)$ \\
\hline Hybrid Ivor-Lewis & $1(8)$ \\
\hline \multicolumn{2}{|l|}{ Anastomosis, $N(\%)$} \\
\hline Cervical, sutured EtE & $1(8)$ \\
\hline Intrathoracic, stapled EtS & $11(92)$ \\
\hline Intraoperative pyloric drainage procedures, $N(\%)$ & $0(-)$ \\
\hline Intraoperative complications, $N(\%)$ & $0(-)$ \\
\hline \multicolumn{2}{|l|}{ Postoperative complications, $N(\%)$} \\
\hline Pneumonia & $1(8)$ \\
\hline CVA & $1(8)$ \\
\hline Anastomotic leakage & $0(-)$ \\
\hline \multicolumn{2}{|l|}{ Reason for inclusion, $N(\%)$} \\
\hline NGT production & $8(67)$ \\
\hline Over $50 \%$ gastric tube distension & $4(33)$ \\
\hline
\end{tabular}

Percentages may not add up to $100 \%$ due to rounding. ASA, American Society of Anesthesiologists; BMI, body mass index, EtE, end to end; $\mathrm{EtS}$, end to side; $\mathrm{GE}$, gastroesophageal; IQR, interquartile range; $\mathrm{MI}$, minimally invasive; $\mathrm{SD}$, standard deviation.

sea, vomiting, or lateral thoracic pain and 3 patients (25\%) due to a $>50 \%$ increase in gastric tube diameter. Following balloon dilation, the median time to discharge was 4 days (IQR 2-6) with oral intake supplemented with enteral nutrition through the jejunostomy tube in 9 patients (75\%). 
Table 3. Outcomes of endoscopic pneumatic pyloric dilation in delayed gastric tube emptying after esophagectomy

\begin{tabular}{|c|c|}
\hline Variable & Outcome $(N=12)$ \\
\hline \multicolumn{2}{|l|}{ Procedure } \\
\hline Interval from surgery to balloon dilation days, median (IQR) & $12(9-15)$ \\
\hline \multicolumn{2}{|l|}{ Outcomes after procedure } \\
\hline \multicolumn{2}{|l|}{ Gastric tube dilatation, $N(\%)$} \\
\hline Persisting & $5(42)$ \\
\hline Diminishing & $5(42)$ \\
\hline None & $1(8)$ \\
\hline No X-ray performed & $1(8)$ \\
\hline \multicolumn{2}{|l|}{ Successful treatment, $N(\%)$} \\
\hline Clinical & $7(58)$ \\
\hline Technical & $12(100)$ \\
\hline \multicolumn{2}{|l|}{ Reason for replacement of NGT, $N(\%)$} \\
\hline Liquid level in gastric tube & $0(-)$ \\
\hline Gastric tube diameter increase (>50\%) & $3(25)$ \\
\hline Nausea, vomiting, pain & $2(17)$ \\
\hline Total & $5(42)$ \\
\hline Total length of stay, median (IQR), days & $15(12-16)$ \\
\hline Readmission, $N(\%)$ & $1(8)$ \\
\hline 90-day mortality, $N$ (\%) & $0(-)$ \\
\hline Hospital stay after intervention, median (IQR), days & $4(2-6)$ \\
\hline \multicolumn{2}{|l|}{ Diet at day of discharge, $N(\%)$} \\
\hline Liquid & $6(50)$ \\
\hline Nil per mouth & $3(25)$ \\
\hline Soft solid & $3(25)$ \\
\hline \multicolumn{2}{|l|}{ Reflux symptoms, $N$ (\%) } \\
\hline Yes & $4(33)$ \\
\hline \multicolumn{2}{|l|}{ Long-term results } \\
\hline \multicolumn{2}{|l|}{ Diet at 3 months, $N(\%)$} \\
\hline Oral & $8(67)$ \\
\hline Oral + enteral tube feeding & $4(33)$ \\
\hline Enteral tube feeding & $0(-)$ \\
\hline \multicolumn{2}{|l|}{ Diet at 6 months, $N(\%)$} \\
\hline Oral & $12(100)$ \\
\hline Oral + enteral tube feeding & $0(-)$ \\
\hline Follow-up, median (IQR), months & $10(6-27)$ \\
\hline \multicolumn{2}{|l|}{ Complete oral diet at last follow-up, $N(\%)$} \\
\hline Yes & $12(100)$ \\
\hline
\end{tabular}

Treatment was a clinical failure in 5 patients (42\%). These 5 patients spent a median of 2 days before the nasogastric tube could be removed, and oral intake was resumed. Three (25\%) patients with clinically failed treatment were discharged after removing the nasogastric tube with a nil-per-mouth regimen and enteral tube feeding through a jejunostomy tube. The remaining 2 (17\%) patients with clinically failed treatment were discharged with a nasogastric tube, nil-per-mouth regimen, and enteral tube feeding through a jejunostomy tube.
The median total hospital stay was 15 days (IQR $12-$ 16), compared to the median hospital stay of 11 days (IQR 9-17) in the total of 235 patients that underwent an esophagectomy without delayed postoperative gastric emptying in the same period. At 3 months after discharge, all 12 patients had oral intake, but in 4 patients (33\%), the total caloric intake was judged as insufficient by the dietician, and supplementary feeding through a jejunostomy tube was needed.

At 6 months after discharge, 1 patient (8\%) had died due to recurrent disease. At 6-month follow-up, there was no 
clinical evidence of delayed gastric emptying in any of the remaining 11 patients, and all patients had complete oral intake. The median follow-up was 10 months (IQR 6-27).

\section{Discussion}

The current study investigated the safety and efficacy of endoscopic pneumatic balloon dilation in the early postoperative in-hospital phase in patients with early postoperative delayed gastric emptying after esophagectomy with gastric tube reconstruction. In our population, we found an incidence of $6 \%$. After pyloric dilation, $58 \%$ of patients were able to expand oral intake without replacement of a nasogastric tube. No adverse events occurred.

Various definitions of delayed gastric emptying are used in scientific publications and literature, with an incidence varying between 11 and 50\% [3-7]. The use of different definitions by different authors renders the literature difficult to compare. The low incidence of delayed postoperative gastric emptying in our cohort may be caused by several factors. One reason might be that we only included patients with delayed gastric emptying occurring 8-10 days postoperatively. We were interested in this timeframe specifically since an earlier intervention might increase the risk of anastomotic complications. Additionally, the strict definition of this complication combined with a procedure performed before day 11 might have caused a lower incidence than found in the literature. Even though only $6 \%$ of our patients fit the criteria of this study, all of these patients had serious complaints. Expanding the inclusion criteria would defeat the purpose of this study.

The literature contains various publications describing uncontrolled series showing promising results for endoscopic balloon dilation $[4,7,18,19]$, with success rates varying between 19 and $95 \%$. The best results are seen in patients with postoperative delayed gastric emptying occurring within 3 months postoperatively $[4,17,19]$. The current study is the first publication of dilation in the early postoperative period, and our results fall within this wide range. However, where earlier studies [18] reported re-dilation rates of up to $53 \%$ at a median of 13 days (range 4-110 days) after the initial dilation, patients in our cohort did not undergo re-dilations.

A possible explanation for the variation in described success rates is the balloon size. An earlier study by Maus et al. [18] has shown that dilation using a 30-mm balloon leads to a lower re-dilation rate in postoperative pyloric balloon dilation: $20 \%$ after $30-\mathrm{mm}$ balloon versus $56 \%$ after using a $20-\mathrm{mm}$ balloon. The inflation time at maxi-

Pyloric Dilation after Esophagectomy mal pressure (2-3 min) was longer compared to our cohort (1 $\mathrm{min})$, but there are no clinical studies available comparing inflation times. It is also worth noting that Maus et al. [18] reported that these re-dilations occurred at a median of 13 days (range 4-110 days) after the initial dilation; none of our patients required a re-dilation in this period. One possible explanation for this discrepancy is the longer and vastly wider timeframe of the time to initial dilation in the study; median 19.5 days (range 6-181 days).

Outpatient treatment of delayed gastric conduit emptying is widely utilized; however, outpatient treatment means that patients spend the interval between discharge and the eventual intervention without oral intake, potential enteral tube feeding, and a nasogastric tube with accompanying symptoms. One could argue that an initial wait-and-see policy saves a portion of patients from an intervention, allowing for spontaneous resolution of the delayed gastric tube emptying. It is our opinion that the additional waiting period with accompanying symptoms such as loss of appetite, dysphagia, nausea, reflux, and regurgitation would call for an earlier intervention.

The current study has several limitations. Most importantly, no control group was used. All patients with early postoperative delayed gastric emptying during the inclusion period have been included in this prospective cohort. Identifying a control cohort in our prospective research database was not possible since data regarding the inclusion criteria (X-ray results and NGT production) were not routinely recorded. Including patients from earlier cohorts would lead to bias due to greatly varying treatment protocols. Additionally, one of the conditions for reinserting a nasogastric tube and defining the treatment as a failure was a more aggressive indication of $>50 \%$ increase in diameter of the gastric tube. Due to the nature of a chest X-ray, measuring an increase in diameter is difficult, potentially causing bias. This bias could have led to an overestimation of gastric tube emptying in the $4 \mathrm{pa}$ tients that were included based on X-ray characteristics only. Last, successful treatment was defined using clinical measures without objective confirmation. It is worth noting that there is no internationally defined standard regarding the normal diameter of a gastric tube reconstruction. In the current study, we chose not to include re-dilations in the protocol, in anticipation of the results of this study. In future studies, we will certainly consider re-dilations as a part of the treatment protocol. We believe further studies are indicated, but given the low incidence in the present study as well as the moderate success rate, an RCT does not seem feasible. 


\section{Conclusion}

Endoscopic pneumatic balloon dilation of the pylorus is a safe and feasible therapy for early postoperative delayed gastric emptying after esophagectomy with a success rate of $58 \%$ in this small series. At 3 months after discharge, all 12 patients had at least partial oral intake.

\section{Statement of Ethics}

This study was conducted in accordance with the Declaration of Helsinki (as revised in 2013). The study was approved by the Medical Ethics Review Committee of the Amsterdam University Medical Center (No. W16_134 \# 16.155), and informed consent requirement was waived for this study.

\section{Conflict of Interest Statement}

V.B.H. declares a research grant from Olympus and Stryker, in addition to consulting fees from Johnson and Johnson and Medtronic. P.F. declares consulting fees from Olympus, Cook, and Ethicon. The other authors have no conflicts of interest to declare.

\section{Funding Sources}

This study did not receive any funding.

\section{Author Contributions}

All authors contributed to study conception and design. Mertens and Gooszen contributed to administrative support. Fockens, Voermans, Gisbertz, Bredenoord, and Van Berge Henegouwen contributed to provision of study materials or patients. Mertens and Gooszen contributed to collection and assembly of data. All authors contributed to data analysis and interpretation, manuscript writing, and final approval of the manuscript. The authors are accountable for all aspects of the work in ensuring that questions related to the accuracy or integrity of any part of the work are appropriately investigated and resolved.

\section{Data Availability Statement}

The data that support the findings of this study are not publicly available due to the small number of patients, since the data could be traced back to individual patients. Data requests can be sent to the corresponding author (V.B.H.).

\section{References}

1 Anandavadivelan P, Wikman A, Johar A, Lagergren $\mathrm{P}$. Impact of weight loss and eating difficulties on health-related quality of life up to 10 years after oesophagectomy for cancer. Br J Surg. 2018 Mar; 105(4):410-8.

2 Deldycke A, Van Daele E, Ceelen W, Van Nieuwenhove Y, Pattyn P. Functional outcome after ivor lewis esophagectomy for cancer. J Surg Oncol. 2016 Jan;113(1):24-8.

3 Benedix F, Willems T, Kropf S, Schubert D, Stübs P, Wolff S. Risk factors for delayed gastric emptying after esophagectomy. Langenbecks Arch Surg. 2017 May;402(3):547-54.

4 Li B, Zhang J-H, Wang C, Song T-N, Wang Z-Q, Gou Y-J, et al. Delayed gastric emptying after esophagectomy for malignancy. J Laparoendosc Adv Surg Tech A. 2014 May;24(5):306-11.

5 Fritz S, Feilhauer K, Schaudt A, Killguss H, Esianu E, Hennig R, et al. Pylorus drainage procedures in thoracoabdominal esophagectomy: a single-center experience and review of the literature. BMC Surg. 2018;18(1):13-8.

6 Akkerman RD, Haverkamp L, van Hillegersberg $\mathrm{R}$, Ruurda JP. Surgical techniques to prevent delayed gastric emptying after esophagectomy with gastric interposition: a systematic review. Ann Thorac Surg. 2014 Oct;98(4):1512-9.

7 Datta J, Williams NN, Conway RG, Dempsey DT, Morris JB. Rescue pyloroplasty for refractory delayed gastric emptying following esophagectomy. Surgery. 2014;156:290-7.
8 Deng B, Tan QY, Jiang YG, Zhao YP, Zhou $J H$, Chen GC, et al. Prevention of early delayed gastric emptying after high-level esophagogastrostomy by "pyloric digital fracture". World J Surg. 2010 Dec;34(12):2837-43.

9 Lanuti M, de Delva PE, Wright CD, Gaissert HA, Wain JC, Donahue DM, et al. Postesophagectomy gastric outlet obstruction: role of pyloromyotomy and management with endoscopic pyloric dilatation. Eur J Cardiothorac Surg. 2007;31(2):149-53.

10 Arya S, Markar SR, Karthikesalingam A, Hanna GB. The impact of pyloric drainage on clinical outcome following esophagectomy: a systematic review. Dis Esophagus. 2015 May; 28(4):326-35.

11 Antonoff MB, Puri V, Meyers BF, Baumgartner K, Bell JM, Broderick S, et al. Comparison of pyloric intervention strategies at the time of esophagectomy: is more better; 2014.

12 Tham JC, Nixon M, Ariyarathenam AV, Humphreys L, Berrisford R, Wheatley T, et al. Intraoperative pyloric botulinum toxin injection during Ivor-Lewis gastroesophagectomy to prevent delayed gastric emptying. Dis Esophagus. 2019 Jun;32(6):1-6.

13 Camilleri M, Parkman HP, Shafi MA, Abell TL, Gerson L. Clinical guideline: management of gastroparesis. Am J Gastroenterol. 2013;108(1):18-38.
14 Bagheri R, Fattahi SH, Haghi SZ, Aryana K, Aryanniya A, Akhlaghi S, et al. Botulinum toxin for prevention of delayed gastric emptying after esophagectomy. Asian Cardiovasc Thorac Ann. 2013 Dec;21(6):689-92.

15 Eldaif SM, Lee R, Adams KN, Kilgo PD, Gruszynski MA, Force SD, et al. Intrapyloric botulinum injection increases postoperative esophagectomy complications. Ann Thorac Surg. 2014 Jun;97(6):1959-5.

16 Kim JH, Lee HS, Kim MS, Lee JM, Kim SK, Zo JI. Balloon dilatation of the pylorus for delayed gastric emptying after esophagectomy. Eur J Cardiothorac Surg. 2008;33(6):1105-11.

17 Lanuti M, Dedelva P, Morse CR, Wright CD, Wain JC, Gaissert HA, et al. Management of delayed gastric emptying after esophagectomy with endoscopic balloon dilatation of the pylorus. Ann Thorac Surg. 2011 Apr;91(4): 1019-24.

18 Maus MK, Leers J, Herbold T, Bludau M, Chon SH, Kleinert R, et al. Gastric. Outlet obstruction after esophagectomy: retrospective analysis of the effectiveness and safety of postoperative endoscopic pyloric dilatation. World J Surg. 2016 Oct;40(10): 2405-11.

19 Swanson EW, Swanson SJ, Swanson RS. Endoscopic pyloric balloon dilatation obviates the need for pyloroplasty at esophagectomy. Surg Endosc. 2012;26(7):20238. 